\title{
RETRACTED ARTICLE: miR-598 induces replicative senescence in human adipose tissue-derived mesenchymal stem cells via silent information regulator 1
}

\author{
Keun Koo Shin • Yeon Jeong Kim • \\ Chang Pyo Hong $\cdot$ Ji Won Yang $\cdot$ Yong Chan Bae \\ Jin Sup Jung
}

Received: 16 January 2012/ Accepted: 3 May 2012/Published online: 13 May 2012

(C) Springer Science+Business Media, LLC. 2012

This article is being retracted at the request of the author due to carelessness in including some of the figures, which were published elsewhere.

K. K. Shin · Y. J. Kim · C. P. Hong · J. W. Yang ·

J. S. Jung $(\bowtie)$

Department of Physiology, School of Medicine, Pusan National

University, Beomeo-ri, Mulgeum-eup, Yangsan-si,

Gyeongsangnam-do 626-870, Korea

e-mail: jsjung@pusan.ac.kr

K. K. Shin · Y. J. Kim · C. P. Hong · J. W. Yang · J. S. Jung

Medical Research Center for Ischemic Tissue Engineering,

Pusan National University, Yangsan, Gyeongnam 626-870,

Korea

K. K. Shin · C. P. Hong · J. S. Jung

BK21 Medical Science Education Center, School of Medicine, Pusan National University, Yangsan, Gyeongnam 626-870,

Korea

Y. C. Bae

Department of Plastic Surgery, School of Medicine, Pusan

National University, Pusan 602-739, Korea

J. S. Jung

Medical Research Institute, Pusan National University, Pusan

602-739, Korea 